Purdue University Purdue e-Pubs

ECE Technical Reports

Electrical and Computer Engineering

$12-1-2004$

\title{
Geographic Routing in the Presence of Location Errors
}

Sungoh Kwon

Ness B. Shroff

Follow this and additional works at: http://docs.lib.purdue.edu/ecetr

Kwon, Sungoh and Shroff, Ness B. , "Geographic Routing in the Presence of Location Errors" (2004). ECE Technical Reports. Paper 130.

http://docs.lib.purdue.edu/ecetr/130

This document has been made available through Purdue e-Pubs, a service of the Purdue University Libraries. Please contact epubs@purdue.edu for additional information. 


\section{GEOGRAPHIC ROUTING IN THE PRESENCE OF LOCATION ERRORS}

SUNGOH KWON

NESS B. SHROFF

TR-ECE 04-10

DECEMBER 2004

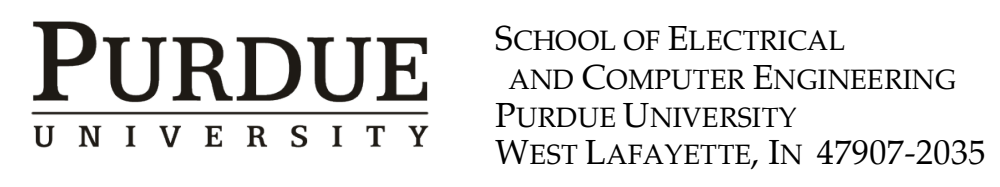




\title{
Geographic Routing in the Presence of Location Errors
}

\author{
Sungoh Kwon and Ness B. Shroff \\ Center for Wireless Systems and Applications (CWSA) \\ School of Electrical and Computer Engineering \\ 465 Northwestern Ave. \\ Purdue University \\ West Lafayette, IN 47907-2035 ${ }^{1}$
}

\footnotetext{
${ }^{1}$ This work has been partially supported by the NSF grant ANI-0099137 and the Indiana 21st Century Fund 651-1285-074.
} 


\section{Contents}

1 Introduction 1

2 The Impact of Location Error on Geographic Routing Performance 4

2.1 Error Modeling . . . . . . . . . . . . . . . . . . 4

2.2 Transmission Failure Probability $\ldots \ldots \ldots \ldots$

2.3 Backward Progress Probability . . . . . . . . . . . . 7

2.4 Impact of Location Error on Geographic Routing . . . . . . . . 8

3 Algorithm 11

3.1 Objective Function $\ldots \ldots \ldots \ldots \ldots \ldots$

3.2 Properties of the Algorithm . . . . . . . . . . . . . . 14

4 Simulation Results 18

4.1 The Performance of MER when the Distribution of the Location

Error is known . . . . . . . . . . . . . . . . . . . . 19

4.2 Robustness to Estimation Error . . . . . . . . . . . . . . . 20

4.3 Mobile Environment . . . . . . . . . . . . . . . . . 24

4.4 Discussion . . . . . . . . . . . . . . . . . 27

5 Conclusions $\quad 28$ 


\section{List of Tables}

4.1 Scenarios for simulations: A, N, R and $\sigma$ represent a deployed area, the number of deployed nodes, the transmission range of nodes and the standard deviation of location errors, respectively $\quad 19$

4.2 The comparison of the number of hops in Scenario 1. . . . . . . . 22

4.3 The comparison of the number of hops in Scenario 2. . . . . . . 22

4.4 The comparison of the number of hops in Scenario 3. . . . . . . . 22 


\section{List of Figures}

1.1 The examples of forwarding schemes when node $\mathrm{S}$ with transmission range $\mathrm{R}$ have a packet to send to node $\mathrm{D}$ : compass rout$\operatorname{ing}(\mathrm{C}), \mathrm{GRS}(\mathrm{G}), \operatorname{MFR}(\mathrm{M})$ and $\mathrm{NFP}(\mathrm{N}) \ldots . . . . . .2$

2.1 Location error modeling when node $i$ with the transmission range $r_{i}$ has a packet to send the destination node $\mathrm{d} . \ldots . . . .5$

2.2 Failure probabilities versus the standard deviation of location errors when $\eta_{i j}=0.8 r_{i}$. The standard deviation of location errors is normalized by the transmission range $r_{i} \ldots \ldots \ldots 9$

2.3 Failure probabilities versus the location of node $j$ when the standard deviation of location errors is $0.1 r_{i}$. The location of the chosen node is normalized by the transmission range $r_{i}$. . . . . 9

2.4 The modified message format for geographic routing. . . . . . . . 10

3.1 The expected progress of node $j$ with respect to node $i$ that has the transmission range $r_{i}$ and is located at $O \ldots \ldots 12$

3.2 The revenue versus the distance from the sender when the transmission range is $1 . \ldots \ldots . . \ldots 16$

3.3 The maximum revenue versus the standard deviation normalized by the transmission range. . . . . . . . . . . . . . 17

3.4 The maximizer versus the standard deviation when the transmission range is $1 . \ldots \ldots \ldots$. . . . . . . . . . . . 17

4.1 The performance comparison of forwarding schemes in Scenario 1. 20

4.2 The performance comparison of forwarding schemes in Scenario 2. 21

4.3 The performance comparison of forwarding schemes in Scenario 3. 21

4.4 The performance of forwarding schemes versus the number of nodes with different error distributions and the fixed transmission range. ........................ 23 
4.5 The performance of forwarding schemes versus the parameter error in estimation with the fixed number of nodes and the fixed transmission range. . . . . . . . . . . . . . . . . . 24

4.6 The performance of forwarding schemes versus the number of nodes with parameter errors in location estimation. When the estimated standard deviation of location errors is $5 \mathrm{~m}$, the standard deviation $\sigma_{r}$ of real location errors varies from $1 \mathrm{~m}$ to $20 \mathrm{~m}$. . . . 25

4.7 The performance of forwarding schemes versus the number of nodes with Gaussian location errors and the RWP mobility model. 26 


\begin{abstract}
In this paper, we propose a new geographic routing algorithm that alleviates the effect of location errors on routing in wireless ad hoc networks. In most previous work, geographic routing has been studied assuming perfect location information. However, in practice there could be significant errors in obtaining location estimates, even when nodes use GPS, hence existing geographic routing schemes will need to be appropriately modified. We investigate how such location errors affect the performance of geographic routing strategies. We incorporate location errors into our objective function by considering both transmission failures and backward progress. Each node then forwards packets to the node that maximizes this objective function. We call this strategy Maximum Expectation within transmission Range (MER). Simulation results with MER show that accounting for location errors significantly improves the performance of geographic routing. Our analysis also shows that our algorithm works well up to a critical threshold of error. We also show that MER is robust to the location error model and model parameters. Further, via simulations, we show that in a mobile environment MER performs better than existing approaches.
\end{abstract}




\section{Chapter 1}

\section{Introduction}

Geographic routing for multi-hop wireless networks has become an active area of study over the last few years (e.g., see [1, 2, 3, 4] and the references therein). Given that geographic information is available, geographic routing is appealing because it is simple to carry out and is scalable. However, most work in this area has implicitly assumed that location information available at each node is perfect, while in practice only a rough estimate of this information is available. In this paper, we will show that imperfect location information can lead to substantial degradation in the performance of geographic routing. Further, in this paper, we will also develop a routing scheme that accounts for location errors, and whose performance is robust to such errors.

In geographic routing, each node determines its own location (typically using the Global Positioning System (GPS) [5, 6] or the location sensing techniques $[7,8,9,10])$ and broadcasts its location information to other nodes proactively and periodically. Packet forwarding is accomplished based on the neighbors' locations stored in each node's database (DB) and the destination's location contained in the packet. Packets are typically forwarded using what is commonly referred to as the greedy mode, in which nodes use local information to forward packets towards their destination. If the greedy mode is not successful (i.e., the destination node is not available in the local databases, or a greedy mode forwarding results in a failure), a special routine called a recovery mode is initiated through the entire network to find an appropriate route to the destination. Since the greedy mode uses local information and most packets are forwarded in this mode [11], geographic routing is generally considered to be scalable and applicable to large networks.

Several forwarding schemes have been proposed for the greedy mode $[12,13$, $14,15]$. Figure 1.1 provides an illustration of such schemes when node $\mathrm{S}$ with 


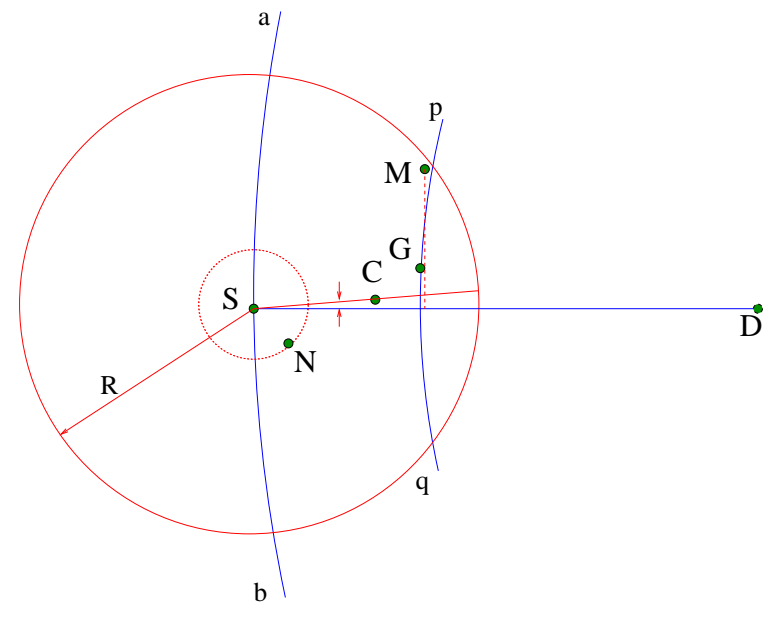

Figure 1.1: The examples of forwarding schemes when node $\mathrm{S}$ with transmission range $R$ have a packet to send to node $D$ : compass routing $(C), \operatorname{GRS}(G), M F R(M)$ and $\mathrm{NFP}(\mathrm{N})$

transmission range $\mathrm{R}$ has a packet to send to node $D$. Arcs $\widehat{a b}$ and $\widehat{p q}$ are centered at node $D$ and with radii $D S$ and $D G$, respectively. When the nodes in the wireless network have a fixed transmission range, the Most Forward within Radius (MFR) scheme [12] and the Greedy Routing Scheme (GRS) [13] have been proposed to minimize the hop count and the energy consumption. MFR forwards a packet to the neighbor (node M in Figure 1.1) that is the farthest from the source in the direction of the destination within the transmission range. GRS selects the closest neighbor (node $\mathrm{G}$ in Figure 1.1) to the destination among neighbors. Since in most cases MFR and GRS provide the same path to the destination [16], we only consider GRS in this paper. When nodes have the ability to control the transmission ranges, the Nearest Forward Progress (NFP) algorithm [14] has been proposed to reduce energy power consumption. NFP chooses the closest neighbor (node $\mathrm{N}$ in Figure 1.1) to the sender within the forward region. Yet another scheme is called compass routing [15]. Compass routing selects that neighbor (node $\mathrm{C}$ in Figure 1.1) which has a minimum angle with respect to the line between the sender and the destination.

Most work on geographic routing assumes that location information received from GPS (or other techniques) is perfect [1, 16, 17, 18, 19, 20]. Effective algorithms in the recovery mode have been addressed in $[1,16,17,18,19]$. Flooding 
based algorithms are proposed for finding alternative paths in $[16,19]$. Face routing (perimeter routing) is also studied for route recovery in [1, 17]. For [20], the authors consider the information discrepancy due to mobility and propose a mobility prediction scheme based on reported location information. However, the location information in [20] is also assumed to be measured perfectly by GPS.

In practice, the received location information is not perfect $[5,10,21]$ and the error in location information degrades the performance of geographic routing [22]. This inaccuracy is caused even when nodes use GPS because of GPS's inherent error in location estimation [6]. This location error could induce a transmission failure and backward progress in the greedy mode. A transmission failure happens when the selected node is out of the transmission range. Backward progress occurs when the chosen node is located farther from the destination than the sender and could cause looping. Loops occur when the selected node is one of the previous senders in the route. Further, it could also result in a local minimum, i.e., there exist solutions but there are no nodes in the forwarding direction. Such failures result in unnecessary transitions from the greedy mode to the recovery mode, which result in an inefficient routing solution $[16,17]$. Even though the greedy mode does not guarantee the delivery of packets, the vast majority of packets are forwarded by the greedy mode [11].

In this paper, we study the impact of these location errors on the performance of geographic routing. We further propose a new routing scheme to improve the performance of geographic routing. We focus on the case when the transmission ranges of nodes are fixed. Using numerical simulations we verify the performance of the proposed algorithm and the robustness of the location error models and model parameters in static wireless networks. We also apply our algorithm to the mobile environment and verify the performance by simulations.

The rest of the paper is organized as follows. In Chapter 2, we study how location errors in geographic routing arise and evaluate the impact of location errors on the performance of the geographic routing. In Chapter 3, we propose a new algorithm for geographic routing in an environment with location errors and analyze properties of the algorithm. In Chapter 4, we use simulation to compare the performance of our scheme with that of known schemes. Chapter 5 concludes this paper. 


\section{Chapter 2}

\section{The Impact of Location Error on Geographic Routing Performance}

In this chapter, we develop a location error model and investigate the impact of location errors on the performance of geographic routing. As mentioned in the introduction, errors in the location information affect the forwarding scheme in the greedy mode, which could cause unnecessary transitions into the recovery mode of the algorithm in order to find an alternative route to the destination. The goal of the greedy mode is to succeed in transmitting packets to a neighbor with forward progress (i.e., the neighbor is closer to the destination). Thus, it is important to account for both transmission failures and backward progress to analyze how the location error affects the performance of geographic routing.

\subsection{Error Modeling}

Location errors occur during the process of estimating the location (via GPS or other techniques) $[5,6,10,21]$. In GPS, the performance of an estimated location depends on the individual environments; the geometry of satellites, location sensing techniques, radio environments, and so on [5]. For example, when GPS uses a single frequency, the root mean square measurement error is typically $6 \mathrm{~m} \mathrm{[6].} \mathrm{On}$ the other hand, the typical measurement error is about $3 \mathrm{~m}$ for a dual-frequency receiver [6]. The geometry of satellites and location sensing techniques are known parameters to the GPS receiver. Other factors are also estimated and adjusted by the GPS receiver.

In this paper we make following assumptions. All nodes are equipped with 


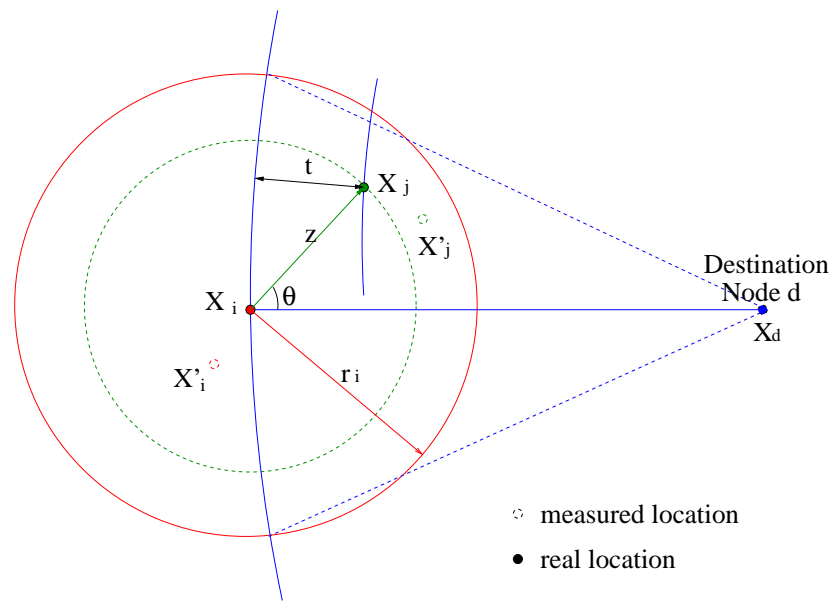

Figure 2.1: Location error modeling when node $i$ with the transmission range $r_{i}$ has a packet to send the destination node $\mathrm{d}$.

GPS to measure their own locations. These locations are proactively broadcasted. The location errors at different nodes are independent. The location error at each node is modeled by a Gaussian distribution ${ }^{1}$ with zero mean and finite standard deviation. The zero-mean assumption implies that, for an given environment, the average of location errors over all nodes is equal to zero, i.e., it follows from the strong law of large numbers [23] that $\lim _{n \rightarrow \infty} \frac{1}{n} \sum_{k=1}^{n} W_{k}$ is equal to zero almost surely, where $W_{i}$ represents a measurement error of node $i$. However, note that for a given environment, the time average of location errors at a single node could be non-zero. Therefore, a scheme that uses simple averaging of samples in time at each node will not be able to overcome the errors.

Let $X_{i}$ be the real position of node $i$ and let $X_{i}^{\prime}$ be its measured position. Then $X_{i}$ can be expressed as $X_{i}=X_{i}^{\prime}+W_{i}$, where $W_{i}$ is a Gaussian random vector with zero mean and standard deviation $\sigma_{i}$.

For convenience, we assign node $i$ to be at the origin and destination node $d$ to be on the $x$-axis, as in Figure 2.1. Let node $j$ be a neighbor of node $i$, and let $z$ be the real distance between the two nodes. Since $X_{i}$ and $X_{j}$ are independent and Gaussian, the probability density function $f_{j}(z, \theta)$ that node $j$ is located at $(z, \theta)$

\footnotetext{
${ }^{1}$ In Chapter 4, we will also study cases when the errors are not Gaussian and their impact on the routing performance.
} 
is

$$
f_{j}(z, \theta)=\frac{z}{2 \pi \sigma_{i j}^{2}} \exp \left(-\frac{z^{2}+\eta_{i j}^{2}}{2 \sigma_{i j}^{2}}\right) \exp (x \cos \theta),
$$

where $\sigma_{i j}$ is the standard deviation of $X_{i}-X_{j}, \theta$ is an angle of $X_{j}$ with respect to $x$-axis, and $\eta_{i j}=\left\|X_{i}^{\prime}-X_{j}^{\prime}\right\|$ as in Figure 2.1. Hence the probability density function $f(z)$ that the distance between two nodes is $z$ is

$$
\begin{aligned}
f_{j}(z) & =\int_{0}^{2 \pi} f_{j}(z, \theta) d \theta \\
& =\frac{z}{\sigma_{i j}^{2}} \exp \left(-\frac{z^{2}+\eta_{i j}^{2}}{2 \sigma_{i j}^{2}}\right) I_{0}\left(\frac{z \eta_{i j}}{\sigma_{i j}^{2}}\right), \text { for } z \geq 0
\end{aligned}
$$

where $I_{0}(x)$ is the modified Bessel function of the first kind and zero order, which is defined by $I_{0}(x)=\frac{1}{2 \pi} \int_{0}^{2 \pi} \exp (x \cos \theta) d \theta$.

\subsection{Transmission Failure Probability}

A packet transmission failure occurs when a chosen node is out of the transmission range of a sender. Most work assumes that the transmission range of each node is perfectly circular and identical so that a neighbor is within the transmission range of a node if and only if the node is located within the transmission range of the neighbor. In practice, nodes have imperfect circular transmission patterns [24] and the transmission ranges deviate from the ideal case [20]. Moreover, a network may be composed of heterogeneous nodes that have different transmission ranges. In these cases, even though a node is located in the transmission range of a neighbor, the neighbor may be out of the transmission range of the node. Such a link is an asymmetric communication link. In the case of asymmetric communication links, transmission failures can happen in the presence of location errors even though each node exactly knows its own transmission range and pattern. In the case when a transmission range is controllable, the adjusted transmission range of a sender can also fail to transmit a packet to its neighbor that is still within the maximum transmission range of the sender.

Assume that node $i$ has a packet to transmit and node $j$ is chosen as the next node. The probability that a packet transmission from node $i$ to node $j$ fails is

$$
\operatorname{Pr}\{\text { transmission failure at node } j\}
$$




$$
\begin{aligned}
& =\operatorname{Pr}\left\{Z>r_{i}\right\} \\
& =\int_{r_{i}}^{\infty} f(z) d z \\
& =\int_{r_{i}}^{\infty} \frac{z}{\sigma_{i j}^{2}} \exp \left(-\frac{z^{2}+\eta_{i j}^{2}}{2 \sigma_{i j}^{2}}\right) I_{0}\left(\frac{z \eta_{i j}}{\sigma_{i j}^{2}}\right) d z \\
& =\int_{\frac{r_{i}}{\sigma_{i j}}}^{\infty} r \exp \left(-\frac{1}{2}\left(r^{2}+\frac{\eta_{i j}^{2}}{\sigma_{i j}^{2}}\right)\right) I_{0}\left(r \frac{\eta_{i j}}{\sigma_{i j}}\right) d r \\
& =Q_{1}\left(\frac{\eta_{i j}}{\sigma_{i j}}, \frac{r_{i}}{\sigma_{i j}}\right),
\end{aligned}
$$

where $Z=\left\|X_{i}-X_{j}\right\|, \sigma_{i j}$ is the standard deviation of $X_{i}-X_{j}, r_{i}$ is the transmission range of node $i, \eta_{i j}=\left\|X_{i}^{\prime}-X_{j}^{\prime}\right\|$, and $Q_{1}(a, b)$ is a Marcum's Q function with $m=1$ defined as in [25].

It follows from Marcum's $\mathrm{Q}$ function with $m=1$ that the transmission failure probability increases when the standard deviation of location errors $\sigma_{i j}$ increases. When $\sigma_{i j}$ is fixed and the chosen node is closer to the edge of the transmission range, the transmission failure probability increases. In other words, given an error environment, a longer transmission range reduces the transmission failure probability.

\subsection{Backward Progress Probability}

Backward progress occurs when a chosen node $j$ is located farther from a destination than the sending node $i$. Note that there may exist a route to the destination even though there is no neighbor in the forward region. This case is typically called a local minimum in the geographic routing literature and cannot be solved by using only greedy mode, so recovery mode is needed. Assume that node $i$ has a packet to transmit and node $j$ is chosen as the next node. For simplicity, in this section, we assume that the destination location $X_{d}$ in the packet has no error ${ }^{2}$.

The probability that the chosen node $j$, such that $\left\|X_{i}^{\prime}-X_{d}\right\| \geq\left\|X_{j}^{\prime}-X_{d}\right\|$, is located behind (is further away from the destination than node $i$ ) the sender $i$ is

\section{$\operatorname{Pr}\{$ backward progress at node $j\}$}

\footnotetext{
${ }^{2}$ The case where the destination location has error can be similarly treated, although the equations become more notationally complex. For the simulations, we assume that the location information of all nodes, including the destination, has errors.
} 


$$
\begin{aligned}
& =\operatorname{Pr}\left\{(z, \theta) \mid\left\|X_{i}-X_{d}\right\| \leq\left\|X_{j}-X_{d}\right\|\right\} \\
& =\int_{\left\{(z, \theta) \mid\left\|X_{i}-X_{d}\right\| \leq\left\|X_{j}-X_{d}\right\|\right\}} f_{j}(z, \theta) d z d \theta \\
& =\int_{\left\{(z, \theta) \mid\left\|X_{i}-X_{d}\right\| \leq\left\|X_{j}-X_{d}\right\|\right\}} \frac{z}{2 \pi \sigma_{i j}^{2}} \times \exp \left(-\frac{z^{2}+\eta_{i j}^{2}}{2 \sigma_{i j}^{2}}\right) \exp (z \cos \theta) d z d \theta,
\end{aligned}
$$

where $\eta_{i j}=\left\|X_{i}^{\prime}-X_{j}^{\prime}\right\|$ and $\sigma_{i j}$ is the standard deviation of $X_{i}-X_{j}$.

In general, the integral above does not reduce to a closed form and must be evaluated numerically. However, if $r_{i}$ is much smaller than the distance between node $i$ and the destination, $\theta$ is close to 0 , we can approximate equation (2.2) as follows:

$$
\operatorname{Pr}\{\text { backward progress at node } j\} \simeq Q\left(\frac{\eta_{i j}}{\sigma_{i j}}\right),
$$

where $\eta_{i j}=\left\|X_{i}^{\prime}-X_{j}^{\prime}\right\|$ and $Q(x)=\frac{1}{\sqrt{2 \pi}} \int_{x}^{\infty} \exp \left(-\frac{x^{2}}{2}\right) d x$.

It follows from the $\mathrm{Q}$ function that the backward progress probability increases when the standard deviation of location errors $\sigma_{i j}$ increases. When $\sigma_{i j}$ is fixed and the chosen node is closer to the sender, the backward progress probability increases.

Figures 2.2 and 2.3 show these two probabilities. In Figure 2.2, we fix the location of node $j$ and show the relationship between the failure probabilities and the standard deviation of the location error. In Figure 2.3, we fix the location error and show the relationship between the failure probabilities and the distance between node $i$ and node $j$. Given the location error, when the chosen node is closer to the sender or the edge of the transmission range, the failure probability increases.

\subsection{Impact of Location Error on Geographic Rout- ing}

All forwarding schemes in geographic routing suffer from the above mentioned failures. GRS (or MFR) selects the closest neighbor to a destination (or the farthest neighbor from the source in the direction of the destination), so the node is more likely close to an edge of a transmission range than any other neighbors. They are susceptible to a transmission failure. NFP chooses a neighbor which is closest to the sender. This scheme is susceptible to backward progress. Compass 


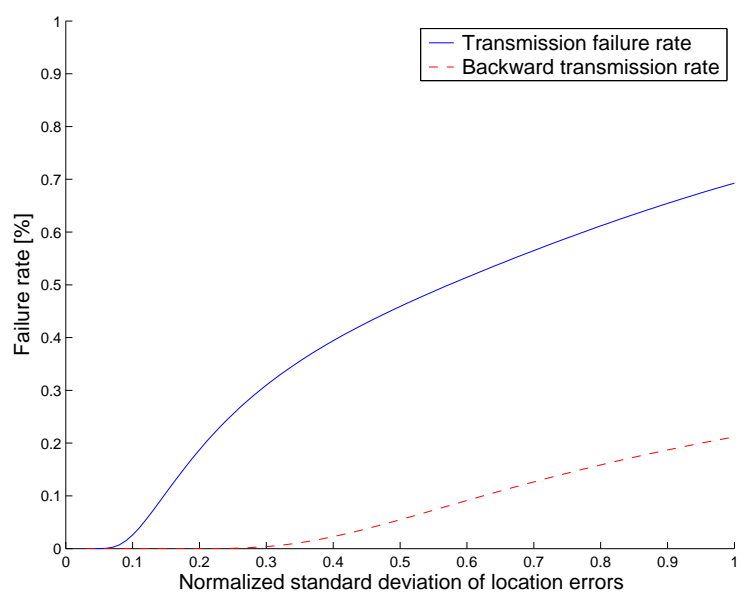

Figure 2.2: Failure probabilities versus the standard deviation of location errors when $\eta_{i j}=0.8 r_{i}$. The standard deviation of location errors is normalized by the transmission range $r_{i}$.

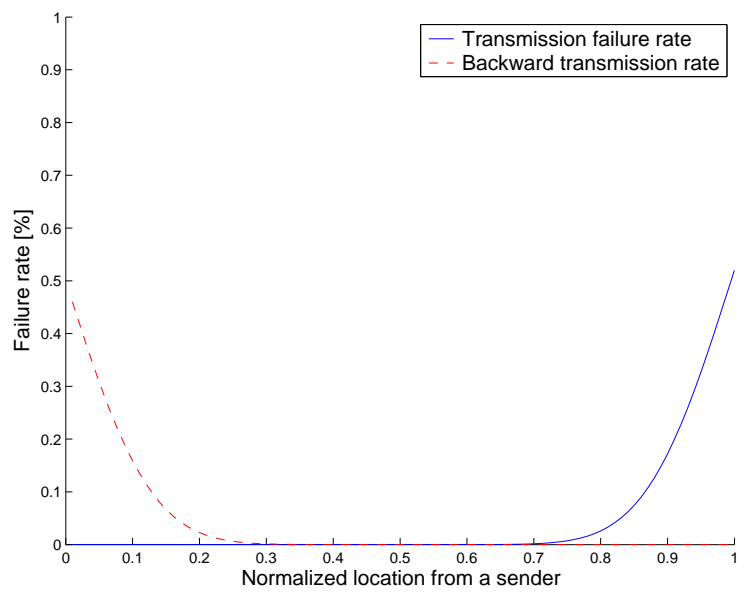

Figure 2.3: Failure probabilities versus the location of node $j$ when the standard deviation of location errors is $0.1 r_{i}$. The location of the chosen node is normalized by the transmission range $r_{i}$. 


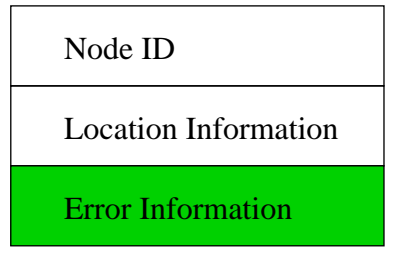

Figure 2.4: The modified message format for geographic routing.

routing does not consider the distance between the sender and the intermediate node, but cares for only the angle with respect to the line between the sender and the destination. Hence, the compass routing is vulnerable to both factors described above. When the number of nodes increases in a given area, a chosen node is closer to the sender or the edge of the transmission range. Hence denser nodes can potentially worsen the performance (as will also be shown via numerical studies in Chapter 4). 


\section{Chapter 3}

\section{Algorithm}

In this chapter we propose a new geographic routing scheme that can mitigate the impact of location errors. Since MFR is similar to GRS in most cases [4], we focus on improving GRS. For ease of illustration, from here on we assume that the transmission range is fixed. However, it should be readily apparent that the methodology can be extended to the case when the transmission range is controllable. Since each node measures its location and estimates its own error characteristic, we attach an error information field in a message for geographic routing, as in Figure 2.4, and announce the statistical characteristics of the location error to neighbors with location information.

\subsection{Objective Function}

Let nodes $i$ and $j$ be located at $X_{i}$ and $X_{j}$, respectively. We assume that $X_{i}^{\prime}$ and $X_{j}^{\prime}$ are the measured locations of nodes $i$ and $j$, respectively. As before, these are expressed as $X_{i}=X_{i}^{\prime}+W_{i}$ and $X_{j}=X_{j}^{\prime}+W_{j}$, where $W_{i}$ and $W_{j}$ are Gaussian random vectors with zero means and standard deviations $\sigma_{i}$ and $\sigma_{j}$, respectively. Then the real position of node $j$ with respect to node $i$ is a Gaussian random vector with mean $X_{j}^{\prime}-X_{i}^{\prime}$ and standard deviation $\sigma_{i j}=\sqrt{\sigma_{i}^{2}+\sigma_{j}^{2}}$. Hence, the probability that node $j$ is located within $u_{j}$ from $X_{j}^{\prime}-X_{i}^{\prime}$ is $\operatorname{Pr}\left\{\left\|X-\left(X_{i}^{\prime}-X_{j}^{\prime}\right)\right\| \leq\right.$ $\left.u_{j}\right\}=1-\exp \left(-\frac{u_{j}^{2}}{2 \sigma_{i j}^{2}}\right)$, where $\mathrm{X}$ is the real position of node $j$ with respect to node $i$.

Fix a sender $i$ and the destination $d$. We define measured progress to node $j$ to be $t_{j}=\left\|X_{d}\right\|-\left\|X_{d}-\left(X_{j}^{\prime}-X_{i}^{\prime}\right)\right\|$ and the measured margin from the boundary 


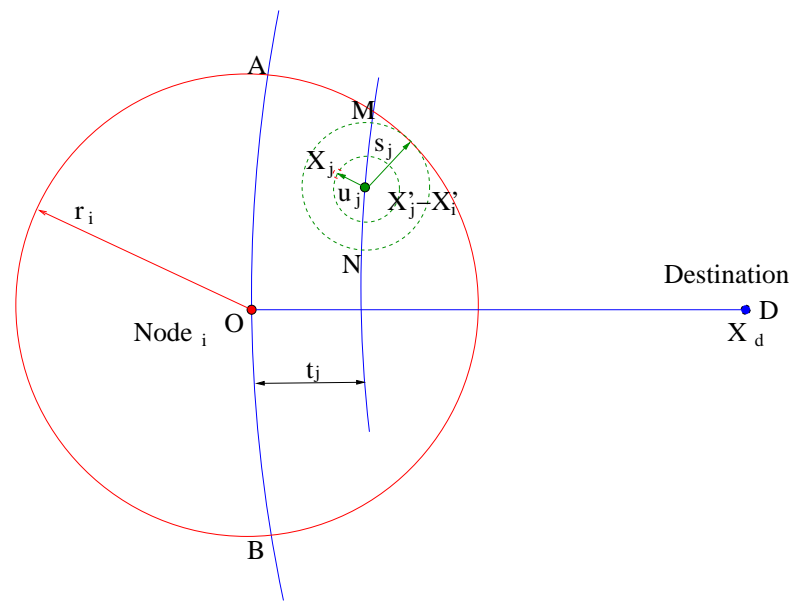

Figure 3.1: The expected progress of node $j$ with respect to node $i$ that has the transmission range $r_{i}$ and is located at $O$.

to be $s_{j}=r_{i}-\left\|X_{i}^{\prime}-X_{j}^{\prime}\right\|$, where $r_{i}$ is the transmission range of node $i$ and $X_{d}$ is the destination position with respect to node $i$. Then we can express GRS in this (location) error-free environment as follows. If $\left\|X_{i}^{\prime}-X_{d}\right\| \leq r_{i}$, choose node $d$. If $\left\|X_{i}^{\prime}-X_{d}\right\|>r_{i}$, choose node $k$ such that

$$
\begin{aligned}
& k=\arg \max _{j \in N_{i}} t_{j} \\
& \text { subject to }\left\|X_{i}^{\prime}-X_{j}^{\prime}\right\| \leq r_{i} \forall j \in N_{i},
\end{aligned}
$$

where $N_{i}$ is the set of neighbors of node $i$.

When we have location errors, instead of using the measured progress $t_{j}$ in (3.1), we propose to use a different metric to determine which neighbor to forward the packets.

We define true progress to node $j$ to be $\tau_{j}=\left\|X_{d}\right\|-\left\|X_{d}-\left(X_{j}-X_{i}^{\prime}\right)\right\|$ when node $j$ is actually located at position $X_{j}$. Then, $\tau_{j}\left(X_{j}\right)$ is a random variable with probability density function $f_{j}\left(X_{j}\right)$. Since the probability density function, $f_{j}\left(X_{j}\right)$, that node $j$ is located at $X_{j}$ is circularly symmetric with respect to the point $X_{j}^{\prime}-X_{i}^{\prime}$, we consider area $A_{j}$ such that $A_{j}=\left\{X \in R^{2} \mid\left\|X-\left(X_{j}^{\prime}-X_{i}^{\prime}\right)\right\| \leq\right.$ $\left.u_{j}\right\}$ and the expected progress of node $j$ over $A_{j}$ as follows.

$$
E\left\{\tau_{j}\left(X_{j}\right) 1_{A_{j}}\right\}=\int_{A_{j}} \tau_{j}\left(X_{j}\right) f_{j}\left(X_{j}\right) d X
$$


If $u_{j}=\infty$, (3.2) becomes the expected progress over the entire domain of $X$. However, if $u_{j}>s_{j}$, node $j$ may be out of the transmission range of node $i$. If $u_{j}>t_{j}$, node $j$ may be located behind node $i$. In order to find a neighbor to be able to successfully transmit to and result in forward progress, we let $u_{j}=\min \left\{s_{j}, t_{j}\right\}$.

For simplicity, we use an approximation of (3.2). In the case of large wireless networks, in most cases $\left\|X_{d}\right\|$ is much greater than $r_{i}$. Then, arc $\overparen{A B}$ and arc $\widehat{M N}$ in Figure 3.1 are nearly straight. Since $f_{j}(z)$ is circularly symmetric, we can simplify (3.2) as follows:

$$
E\left\{\tau_{j}\left(X_{j}\right) 1_{A_{j}}\right\} \simeq t_{j} \int_{A_{j}} f_{j}\left(X_{j}\right) d X_{j}
$$

where $A_{j}=\left\{X \in R^{2}\left\|X-\left(X_{j}^{\prime}-X_{i}^{\prime}\right)\right\| \leq u_{j}\right\}$ for $u=\min \left\{s_{j}, t_{j}\right\}$.

Hence, we define $E_{j}$ as a revenue of node $j$ as follows.

$$
E_{j} \triangleq t_{j} F_{j}\left(u_{j}\right)
$$

where

$$
\begin{aligned}
F_{j}\left(u_{j}\right) & =\operatorname{Pr}\left\{\left\|X-\left(X_{j}^{\prime}-X_{i}^{\prime}\right)\right\| \leq u_{j}\right\} \\
& =1-\exp \left(-\frac{u_{j}^{2}}{2 \sigma_{i j}^{2}}\right)
\end{aligned}
$$

for $u_{j}=\min \left\{s_{j}, t_{j}\right\}$.

Based on the calculated revenue of each node, node $i$ selects the next node which has a Maximum Expectation within transmission Range $r_{i}$ (MER). Our MER algorithm for forwarding packets is given as follows. If $\left\|X_{i}^{\prime}-X_{d}\right\| \leq r_{i}-\delta_{i}$, where $0 \leq \delta_{i}<r_{i}$, choose node $d$. If $\left\|X_{i}^{\prime}-X_{d}\right\|>r_{i}-\delta_{i}$, choose node $k$ such that

$$
\begin{aligned}
& k=\arg \max _{j \in N} E_{j} \\
& \text { subject to }\left\|X_{i}^{\prime}-X_{j}^{\prime}\right\| \leq r_{i} \forall j \in N_{i},
\end{aligned}
$$

where $N_{i}$ is the set of node $i$ 's neighbor nodes stored in the node $i$ 's DB. Here, $\delta_{i}$ is a function of location errors and could be a tuning parameter for specific implementations. $\delta_{i}$ can be simply the standard deviation of location errors or the distance from the maximizer of the objective function to the transmission range edge. In [20], the authors proposed a scheme to improve the forwarding performance as follows: if the destination node exists in the neighbor list, the sender 
forwards the packet to the destination without any effort. However, since in the location error environment the destination may be located out of the transmission range, it is imperative to consider the parameter $\delta_{i}$ to increase the transmission success rate.

\subsection{Properties of the Algorithm}

We now analyze the MER algorithm proposed above. For simplicity, we assume that a selected node is located on the line between the sender and the destination since the node on the line $(\overline{O D}$ in Figure 3.1$)$ is the most likely chosen among neighbors that have the same measured progress. Let the transmission range of the sender $r_{i}$ be 1 . We let $\sigma$ denote as the standard deviation for the overall location error between the sender and the intermediate node. Then, (3.3) becomes

$$
\begin{aligned}
E(t) & =t F(u) \\
& =t\left(1-\exp \left(-\frac{u^{2}}{2 \sigma^{2}}\right)\right)
\end{aligned}
$$

where $t \in(0,1)$ is the position of the selected node and $u=\min \{t, 1-t\}$.

We define an effective search range to be an area from the source to the most likely position chosen by the algorithm and $t_{\max }$ to be a maximizer of $E(t)$ such that $E\left(t_{\max }\right) \geq E(t)$ for $t \in(0,1)$.

Property 1 Let $\sigma \ll 1$, then MER is identical to GRS within an effective search range for a next node. The range is reduced by $\delta$ such that $E^{\prime}(1-\delta)=0$, where $E^{\prime}(t)$ is the derivative of $E(t)$ and the maximizer of $E(t), t_{\max }$, is a decreasing function of $\sigma$.

Proof: In the region of $u=\min \{t, 1-t\} \gg \sqrt{2} \sigma$, from (3.4)

$$
\begin{aligned}
E(t) & =t\left(1-\exp \left(-\frac{u^{2}}{2 \sigma^{2}}\right)\right) \\
& \approx t .
\end{aligned}
$$

Note that (3.5) is equivalent to the objective function of GRS (3.1). Since $E^{\prime}(t)=$ $t F(t)$ is always an increasing function of $t \in(0,0.5)$, we need to consider $t \in$ $(0.5,1)$ to check the maximum of $E(t)$. The derivative of the objective function 
$E^{\prime}(t)$ for $t \in(0.5,1)$ is

$$
\begin{aligned}
E^{\prime}(t) & =F(1-t)-t f(1-t) \\
& <1-\frac{1}{2} f(1-t)
\end{aligned}
$$

Since $\sigma \ll 1$ from the assumption above and $f(t)$ has a maximum $\frac{1}{\sigma} \exp \left(-\frac{1}{2}\right)$ at $t=\sigma, E^{\prime}(t) \ll-1$. The values of $E(t)$ precipitates from a maximum value to zero, and the effective search range reduces to $t_{\max }$ such that $E^{\prime}\left(t_{\max }\right)=0$. In other words, the effective search range is reduced by $\delta$ such that $\delta=1-t_{\max }$. For $\sigma \ll 1$,

$$
\begin{aligned}
E^{\prime}\left(t_{\max }\right) & =F\left(1-t_{\max }\right)-t_{\max } f\left(1-t_{\max }\right)=0 \\
E^{\prime}\left(t_{\max }\right) & \simeq \frac{\left(1-t_{\max }\right)^{2}}{2 \sigma^{2}}-\frac{t_{\max }\left(1-t_{\max }\right)}{\sigma^{2}}\left(1-\frac{\left(1-t_{\max }\right)^{2}}{2 \sigma^{2}}\right) \\
\sigma^{2} & =\frac{t_{\max }^{3}-2 t_{\max }^{2}+t_{\max }}{3 t_{\max }-1}
\end{aligned}
$$

where $t_{\max }$ is the maximizer of (3.4). From (3.6) above, $\sigma$ is a monotonically decreasing function of $t_{\max }$ for $t_{\max } \in(0.5,1)$. Hence $t_{\max }$ is a monotonic decreasing function of $\sigma$.

Property 2 There exists a threshold $\sigma_{\text {th }}$ such that the decrement of $\sigma$ results in an increment of $t_{\max }$ for $\sigma \geq \sigma_{t h}$, but $t_{\max }$ does not depends on $\sigma$ for $\sigma \leq \sigma_{t h}$. The threshold $\sigma_{\text {th }}$ is numerically 0.315 .

Proof: $\quad t F(t)$ is an increasing function for $t \in[0,1] . t F(1-t)$ has a maximizer $t_{\max } \in[0,1]$ and a minimum value 0 at $t=0$ and $1 . t F(1-t)$ increases for $t \in\left[0, t_{\max }\right]$ and decreases for $t \in\left[t_{\max }, 1\right]$ monotonically. It follows from (3.4) that if $t_{\max } \in[0,0.5], \mathrm{E}(\mathrm{t})$ has a maximum at $\mathrm{t}=0.5$ and if $t_{\max } \in[0.5,1)$, $\mathrm{E}(\mathrm{t})$ has a maximum at $\mathrm{t}=t_{\max } \cdot \sigma_{t h}$ is $\sigma$ such that $E^{\prime}\left(\frac{1}{2}\right)=0$. The numerical value of $\sigma_{t h}$ is 0.315 .

Figures 3.2, 3.3 and 3.4 depict how the objective function of the proposed algorithm (MER) works. For these figures, we assume that the selected node is located on $\overline{O D}$ in Figure 3.1. Property 1 shows that MER works identically to GRS except in the outskirts of the transmission range, as shown in Figure 3.2. However, MER requires more hops to route packets from source to destination 


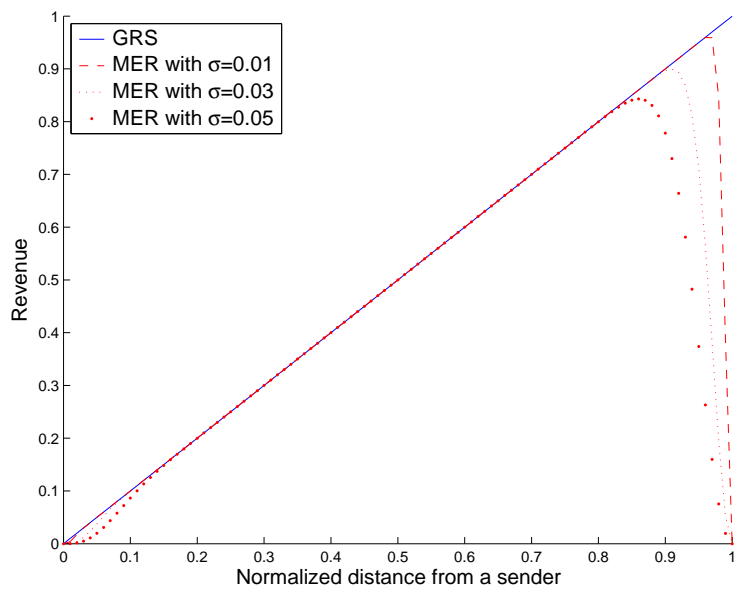

Figure 3.2: The revenue versus the distance from the sender when the transmission range is 1 .

compared with the case of perfect location environment, since the position of the most likely chosen node, $t_{\max }$, decreases due to location error, as in Figures 3.2 and 3.4.

Property 2 gives us some insights on geographic routing in an environment with location errors. If the standard deviation of the location error is greater than some threshold, i.e. $\sigma \geq \sigma_{t h}$, the maximizer of (3.4), $t_{\max }$, decreases when $\sigma$ increases. If $\sigma \leq \sigma_{t h}, t_{\max }$ does not depend on $\sigma$, as in Figure 3.4 and only the maximum revenue decreases as in Figure 3.3. $t_{\max }$ is fixed at 0.5 after $\sigma=$ 0.315 . In this region, increasing $\sigma$ does not affect the selected node, but decreases the revenue. This means that for a standard deviation of error larger than $\sigma_{t h}$, taking error estimates into account does not help in improving the performance of geographic routing. The algorithm is simply reduced to selecting the node that is closest to the middle point of the forward region. Hence, $\sigma_{t h}$ becomes the critical point that determines the utility of the algorithm. For example, when each node is equipped with a GPS receiver that has a standard deviation of $3 \mathrm{~m}$ of the location error, Bluetooth [26] which has a transmission range of $10 \mathrm{~m}$ cannot use geographic routing. However, a geographic routing scheme that incorporates error information (such as MER) is applicable in the case of IEEE 802.11 with a nominal transmission range $250 \mathrm{~m}$. 


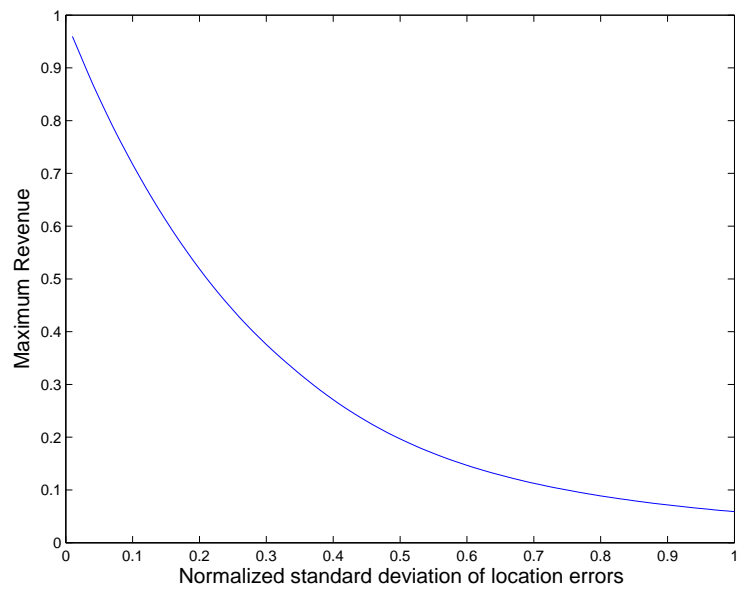

Figure 3.3: The maximum revenue versus the standard deviation normalized by the transmission range.

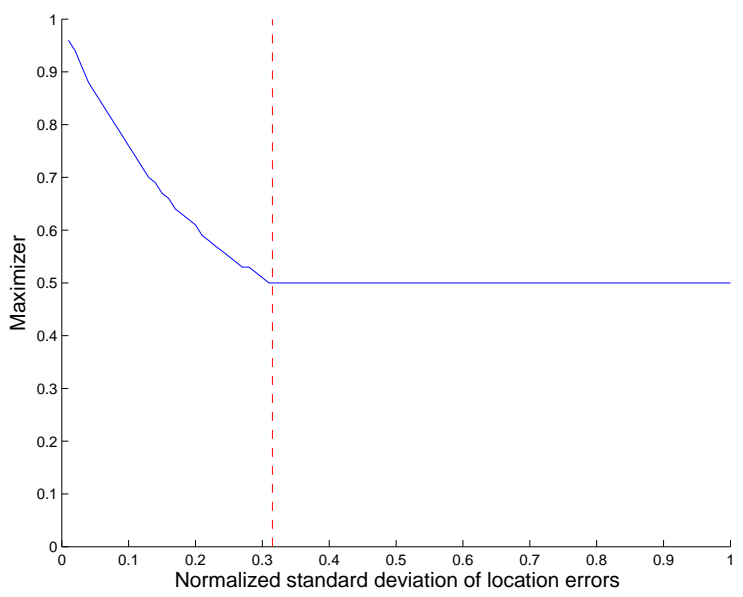

Figure 3.4: The maximizer versus the standard deviation when the transmission range is 1 . 


\section{Chapter 4}

\section{Simulation Results}

In this chapter, we use numerical simulations to verify the performance of the proposed algorithm, MER. First, we compare the performance of MER versus GRS when the parameters of the location error model are known. We further also demonstrate the robustness of the algorithm to changing parameters in the error model. Finally, we study the performance of MER in a mobile environment.

In our simulations we compare two different schemes: GRS and MER. We use the performance of GRS with perfect location information as an upper bound on the routing performance of all schemes in the presence of location errors. For a fair comparison, we do not use retransmissions or algorithms to find alternative routes when packet forwarding failures occur. Packet delivery is said to succeed only if the packet is delivered to the destination by the schemes. In MER, we use the distance from the maximizer of the objective function to the transmission range edge as a tuning parameter $\delta_{i}$. In practice, the wireless environment at each node in the network may be different. For instance, in the case of GPS, each node could receive a different number of satellite signals due to obstacles. Our algorithm described in Chapter 3 can handle such a heterogeneous environment. However, in order to study how system parameters affect the performance of geographic routing in the presence of location errors, for our numerical results we focus on the case when the wireless environment is homogeneous across all nodes. 
Table 4.1: Scenarios for simulations: A, N, R and $\sigma$ represent a deployed area, the number of deployed nodes, the transmission range of nodes and the standard deviation of location errors, respectively

\begin{tabular}{|c|c|c|c|c|}
\hline Scenario & $\mathrm{A}\left(\mathrm{m}^{2}\right)$ & $\mathrm{N}$ & $\mathrm{R}(\mathrm{m})$ & $\sigma(\mathrm{m})$ \\
\hline 1 & $1000 \times 1000$ & 100 & $25 \sim 500$ & $10(2 \% \sim 40 \%)$ \\
\hline 2 & $1000 \times 1000$ & 100 & 250 & $3 \sim 50(1.2 \% \sim 20 \%)$ \\
\hline 3 & $1000 \times 1000$ & $25 \sim 1000$ & 250 & $5(2 \%)$ \\
\hline
\end{tabular}

\subsection{The Performance of MER when the Distribution of the Location Error is known}

We investigate three scenarios as illustrated in Table 4.1. In the first scenario, we deploy 100 nodes in an area of $1000 \times 1000 \mathrm{~m}^{2}$. The standard deviation of the location error for each node is $10 \mathrm{~m}$. We vary the transmission range from $25 \mathrm{~m}$ to $500 \mathrm{~m}$. Figure 4.1 shows that the transmission success rate of MER is close to that of GRS with perfect location information. However, the performance of GRS degrades severely in the presence of location errors. Table 4.2 provides a comparison of the number of hops required for transmission between MER and GRS with perfect location information. Table 4.2 shows that MER with location errors needs some additional hops to reach its destination when compared with GRS with perfect location information. The reason is that the location errors cause MER to reduce its effective search range when forwarding packets to its neighbors, as shown by Property 1 . Hence, MER requires additional hops to deliver packets from source to destination.

In the second scenario, the transmission range is fixed at $250 \mathrm{~m}$, which is the nominal transmission range of IEEE 802.11, and we vary the standard deviation of the location error from $3 \mathrm{~m}(1.2 \%)$ to $50 \mathrm{~m}$ (20\%). As expected, Figure 4.2 shows that MER performs much better than GRS when there are location errors. The performance of GRS starts to degrade when the standard deviation is above $3 \mathrm{~m}(1.2 \%)$. However, the performance of MER does not decrease significantly until the standard deviation is above $12 \mathrm{~m}(4.8 \%)$. As the error increases, the effective search range in MER is further reduced. This reduction of the effective search range decreases the number of neighbor nodes to be selected and degrades the performance. Similarly to Scenario 1, MER with location errors requires additional hops to route packets when compared to the case with perfect information, 


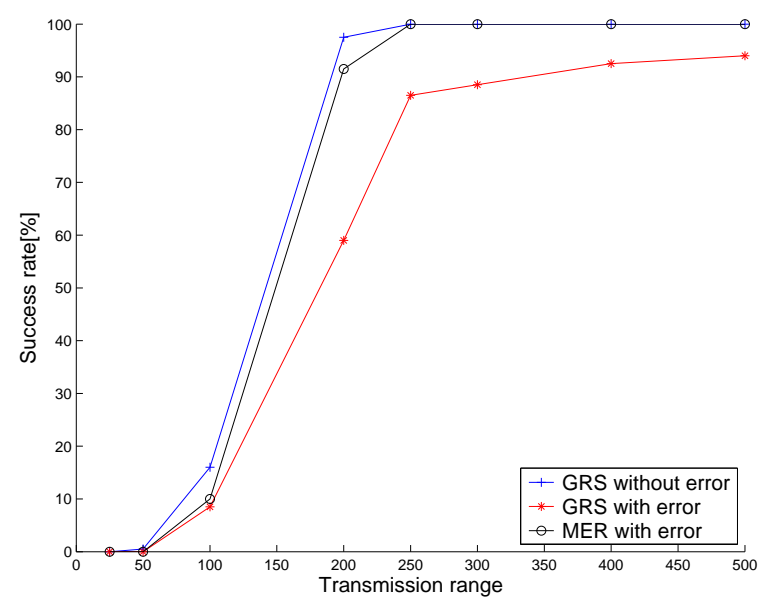

Figure 4.1: The performance comparison of forwarding schemes in Scenario 1.

as shown in Table 4.3.

In the third scenario, we vary the number of deployed nodes from 25 to 1000 . We fix the transmission range at $250 \mathrm{~m}$ and the standard deviation of the location error at $5 \mathrm{~m}$. Figure 4.3 shows that the performance MER is not affected by the number of nodes while the performance of GRS is. The larger density reduces the distance between two adjacent nodes. This reduction in distance means that the selected node is closer to the edge of the transmission range. Hence, transmission failure is more likely to happen in GRS. Similarly to Scenario 1, MER with location errors needs additional hops (Table 4.4) compared to case without location errors, however the success rate does not decrease when the node density of the network is increased. Note that in Table 4.4 that, unlike previous scenarios, the ratio of the additional hops for MER does not change significantly as the number of nodes increases since the maximizer of the objective function depends only on the ratio of the location error to the transmission range.

\subsection{Robustness to Estimation Error}

In Chapter 2, we modeled the location error by a Gaussian error distribution. In practice, the error may not follow a Gaussian distribution and/or the parameters of the model may be incorrect. In this section, we study the robustness of MER with respect to these two kinds of modeling errors: the distribution function and 


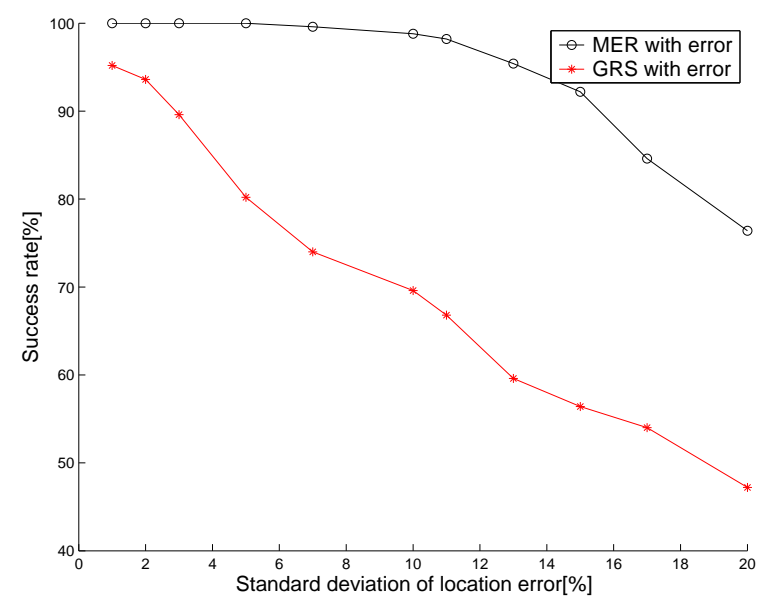

Figure 4.2: The performance comparison of forwarding schemes in Scenario 2.

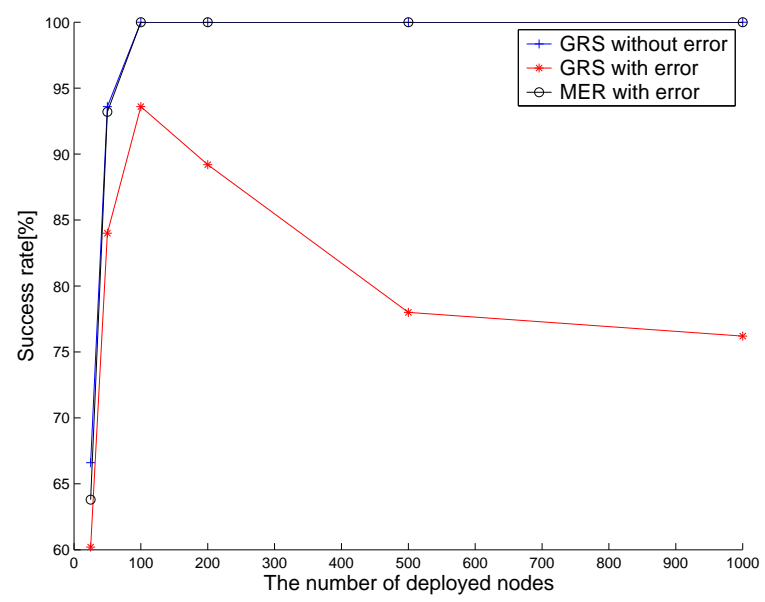

Figure 4.3: The performance comparison of forwarding schemes in Scenario 3. 
Table 4.2: The comparison of the number of hops in Scenario 1.

\begin{tabular}{|c|c|c|c|}
\hline$r_{i}(m)$ & MER with error (a) & GRS without error (b) & $\frac{(a)}{(b)}(\%)$ \\
\hline 250 & 335 & 302 & 117.6 \\
\hline 300 & 280 & 249 & 112.5 \\
\hline 400 & 202 & 191 & 105.8 \\
\hline 500 & 164 & 155 & 105.8 \\
\hline
\end{tabular}

Table 4.3: The comparison of the number of hops in Scenario 2.

\begin{tabular}{|c|c|c|c|}
\hline$\sigma(m)$ & MER with error (a) & GRS without error (b) & $\frac{(a)}{(b)}(\%)$ \\
\hline 3 & 302 & 294 & 102.7 \\
\hline 5 & 308 & 294 & 104.6 \\
\hline 8 & 318 & 294 & 108.2 \\
\hline 18 & 350 & 294 & 119.1 \\
\hline
\end{tabular}

Table 4.4: The comparison of the number of hops in Scenario 3.

\begin{tabular}{|c|c|c|c|}
\hline$N$ & MER with error (a) & GRS without error $(\mathrm{b})$ & $\frac{(a)}{(b)}(\%)$ \\
\hline 100 & 308 & 294 & 104.7 \\
\hline 200 & 302 & 282 & 107.1 \\
\hline 500 & 298 & 277 & 107.6 \\
\hline 1000 & 278 & 260 & 106.9 \\
\hline
\end{tabular}




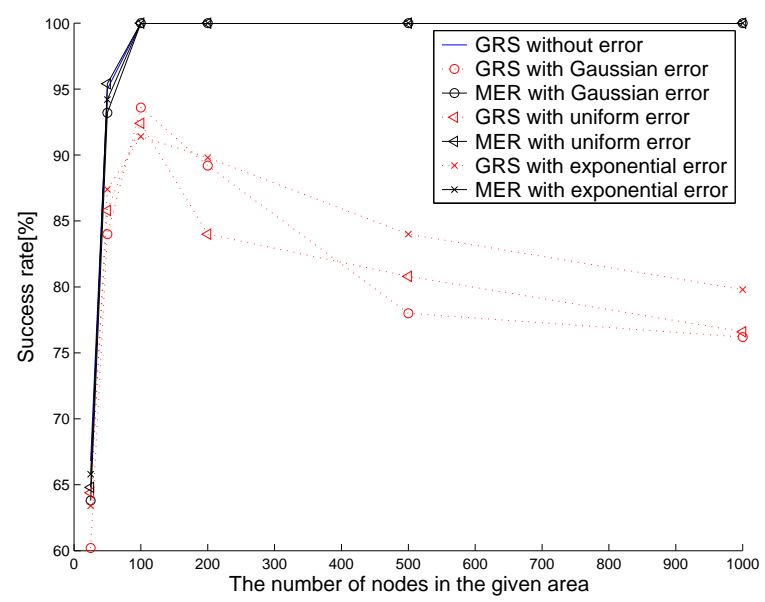

Figure 4.4: The performance of forwarding schemes versus the number of nodes with different error distributions and the fixed transmission range.

parameter error.

In Figure 4.4, we simulate three different location error models: uniformly distributed error, exponentially distributed error, and Gaussian error. However, the MER algorithm always assume a Gaussian model. The transmission range of each node is $250 \mathrm{~m}$ and the standard deviation of the location error is $5 \mathrm{~m}$. The simulation results show that MER is quite robust to different error distribution functions.

In Figures 4.5 and 4.6, the underlying error model is also Gaussian. However the parameter used by MER is different from the true parameter of the underlying model. In the simulation the transmission range of each node is $250 \mathrm{~m}$ and the standard deviation of the location error is assumed to be $5 \mathrm{~m}$. However, the actual standard deviation of the location error is varied from $0 \mathrm{~m}$ from $20 \mathrm{~m}$. The simulation results show that MER has the same performance until the standard deviation deviates from its real value by $8 \mathrm{~m}(160 \%)$ as in Figures 4.5 and 4.6. These results show that MER is robust to the estimation error and outperforms GRS. 


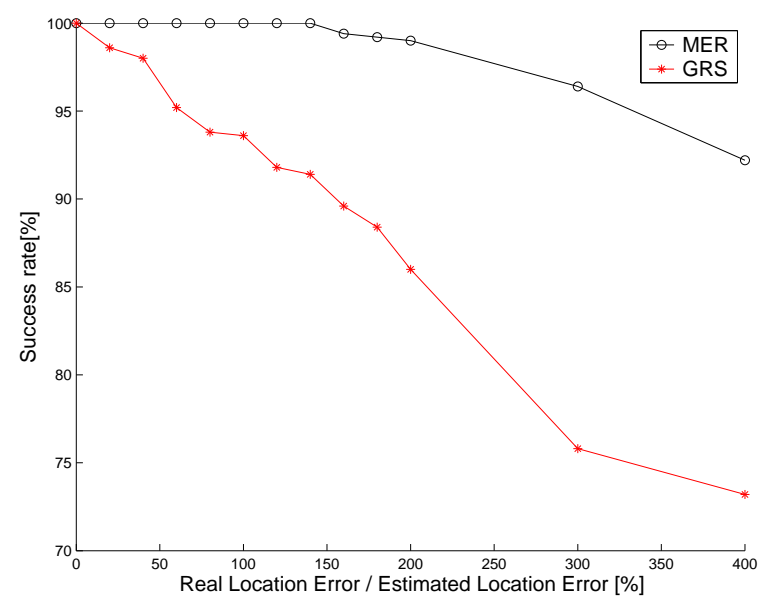

Figure 4.5: The performance of forwarding schemes versus the parameter error in estimation with the fixed number of nodes and the fixed transmission range.

\subsection{Mobile Environment}

In this section, we compare the performance of MER and GRS in a mobile environment. We use the following random way point (RWP) mobility model. Each node chooses a destination in a given area and moves at a constant speed, which is uniformly chosen between 0 and $50 \mathrm{~m} / \mathrm{s}$. The node stays for a pause time which is uniformly distributed between 0 seconds and 30 seconds. Each node broadcasts its own location periodically and proactively. To avoid collisions, the interval of these broadcasts is uniformly chosen from 1 second to 3 seconds, as in [18]. We also set the neighbor timeout interval at 9 seconds. When each node chooses the next node, we use the predicted positions of neighbors at the transmission time similar to $[20,27]$ in order to improve the routing performance. The authors in $[20,27]$ predict the neighbor positions at the transmission time by using two positions reported at two recent times as follows.

$$
X^{(0)}=X^{(1)}+\frac{X^{(1)}-X^{(2)}}{t^{(1)}-t^{(2)}} \times\left(t^{(0)}-t^{(1)}\right),
$$

where $X^{(0)}$ is the predicted position at current time $t^{(0)}, X^{(1)}$ is the reported location at the first recent time $t^{(1)}$, and $X^{(2)}$ is the reported location at the second recent time $t^{(2)}$. The method improves the routing performance by reducing the mobility error when the location information is assumed to be perfect. However, 


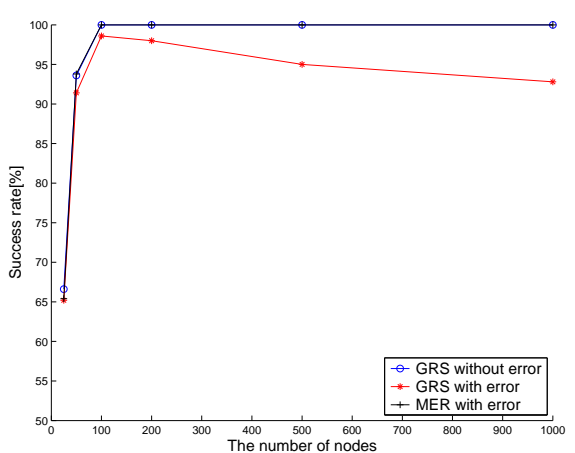

(a) $\sigma_{r}=1 \mathrm{~m}(20 \%)$

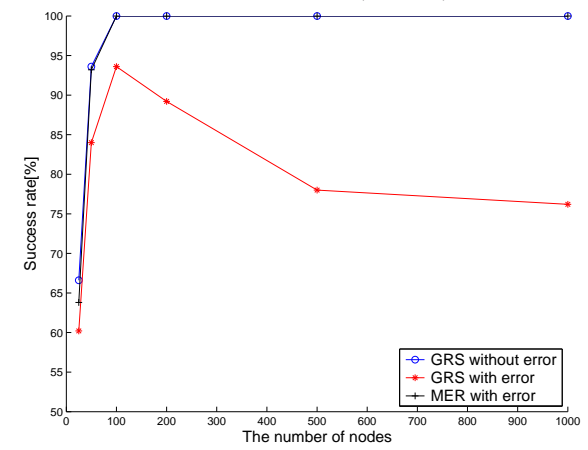

(c) $\sigma_{r}=5 \mathrm{~m}(100 \%)$

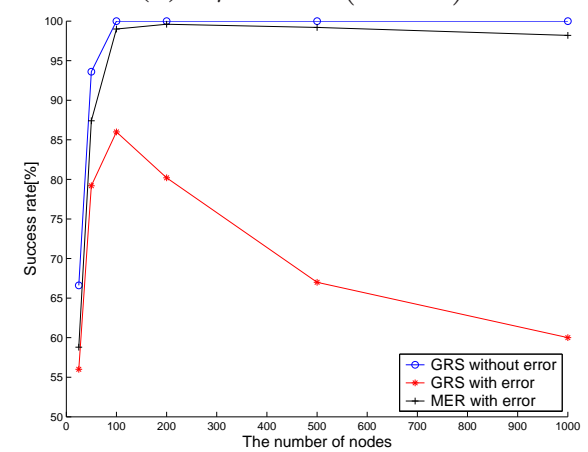

(e) $\sigma_{r}=10 \mathrm{~m}(200 \%)$

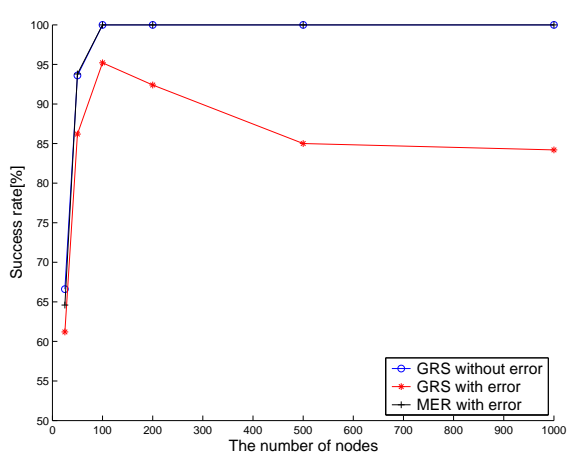

(b) $\sigma_{r}=3 \mathrm{~m}(60 \%)$

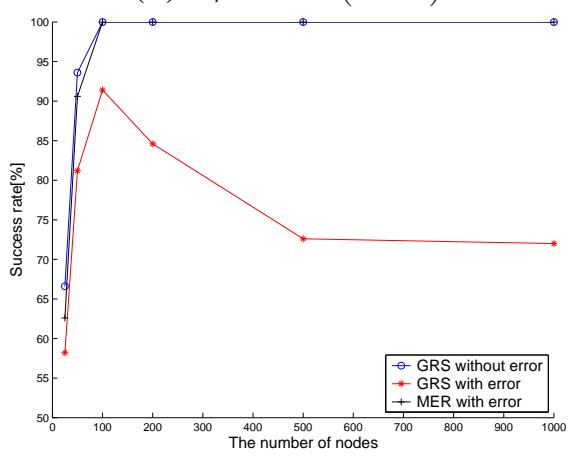

(d) $\sigma_{r}=7 \mathrm{~m}(140 \%)$

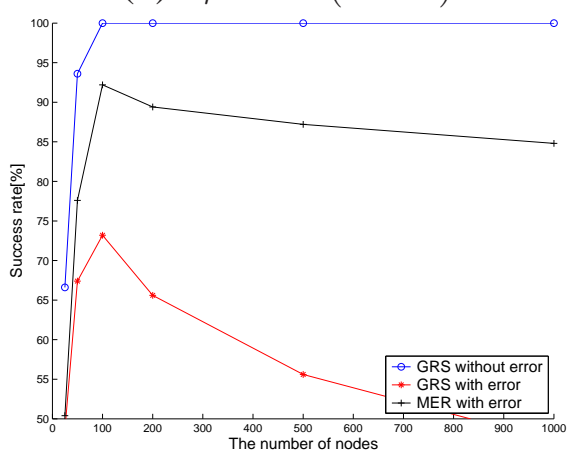

(f) $\sigma_{r}=20 \mathrm{~m}(400 \%)$

Figure 4.6: The performance of forwarding schemes versus the number of nodes with parameter errors in location estimation. When the estimated standard deviation of location errors is $5 \mathrm{~m}$, the standard deviation $\sigma_{r}$ of real location errors varies from $1 \mathrm{~m}$ to $20 \mathrm{~m}$. 


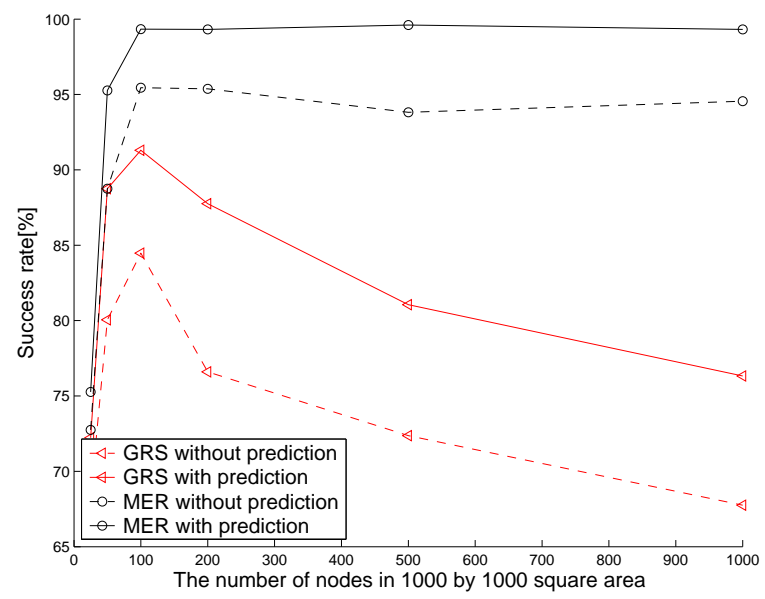

Figure 4.7: The performance of forwarding schemes versus the number of nodes with Gaussian location errors and the RWP mobility model.

this method will accumulate measurement and prediction errors when location information is noisy. Hence, we use the instant velocity, which is available to GPS equipped nodes, since the velocity measured by GPS is considered to be very accurate [6]. The instant velocity is announced with location information. Each node predicts neighbors' positions when forwarding a packet as follows.

$$
X^{(0)}=X^{(1)}+v^{(1)} \times\left(t^{(0)}-t^{(1)}\right),
$$

where $X^{(0)}$ is the predicted position at current time $t^{(0)}, X^{(1)}$ is the reported location at the first recent time $t^{(1)}$, and $v^{(1)}$ is the reported velocity at $t^{(1)}$. Figure 4.7 shows the simulation results when the location error at each node has a Gaussian distribution with standard deviation $5 \mathrm{~m}$ and the transmission range is fixed at $250 \mathrm{~m}$. GRS with the position estimation performs better than GRS without the position estimation, as shown in [20]. MER is slightly affected by mobility but outperforms GRS in all cases. As shown in [11], the performance degradation from mobility can be further improved by reducing time discrepancy. However, since the measurement error of $X^{(1)}$ in (4.2) does not decrease as the time discrepancy reduces. The reduction of the time discrepancy also does not affect the location error of a sender. Hence, any effort to reduce the time discrepancy from mobility cannot help mitigate the impact of measurement errors on geographic routing. 


\subsection{Discussion}

In order to solve the fundamental problem of geographic routing with location errors, in this paper we do not use a protocol specific solution to help mitigate errors. However, such a protocol-specific solution could in certain cases help combat location errors. For example, in the case when nodes are static and have fixed transmission ranges, the transmission failures caused by asymmetric communication links can be reduced by a three-way handshake protocol when nodes join the network. However, the three-way communication does not alleviate backward progress. Even in a static wireless network, such a protocol cannot avoid transmission failures when the transmission range is controllable in the presence of location errors. Further, the solution is not suitable in a dense mobile wireless network, where frequent topology changes may take place. Moreover, in contrast to our proactive announcement (one-way communication) of location information, the three-way communication results in $2 n(n-1)$ overhead messages per time interval in a transmission range, where $n$ represents the number of nodes within a transmission range. The excessive overhead messages may in fact worsen network performance such as throughput. For the above mentioned reasons, in this paper, we do not focus on protocol-specific solutions to alleviate location errors. However, such approaches can be potentially used in conjunction with our method on a case by case basis.

Another feature of our approach is to provide an understanding of the intrinsic performance achievable using geographic routing with location errors. This serves

to provide design guidelines for implementers to choose equipment that provides an appropriate level of accuracy. 


\section{Chapter 5}

\section{Conclusions}

In this paper, we consider the impact of location errors on geographic routing in multi-hop wireless networks. We have shown that location errors can significantly affect the performance of geographic routing. The degradation in the routing performance depends on the transmission range of the sender, error characteristics of the sender and its neighbors, and the deployed density of nodes. We have proposed a new algorithm called MER in order to mitigate the effect of noisy location information by explicitly considering the error probability when making routing decisions. In doing so we find that our algorithm performs quite well, in many cases, even approximating the performance of geographic routing without location errors. We have also used simulations to show that MER is robust to different location error models and errors in model parameters. 


\section{Bibliography}

[1] F. Kuhn, R. Wattenhofer, and A. Zollinger, "Worst-case optimal and averagecase efficient geometric ad-hoc routing," in ACM MobiHoc, 2003, pp. 267278.

[2] M. Mauve, J. Widmer, and H. Hartenstein, "A survey on position-based routing in mobile ad hoc networks," IEEE Network, vol. 15, pp. 30-39, 2001.

[3] S. Ratnasamy, B. Karp, L. Yin, F. Yu, D. Estrin, R. Govindan, and S. Shenker, "GHT-a geographic hash table for data-centric storage," in First ACM International Workshop on Wireless Sensor Networks and their Applications, 2002, pp. 78-87.

[4] I. Stojmenovic, "Position-based routing in ad hoc networks," IEEE Communications Magazine, vol. 40, no. 7, pp. 2-8, July 2002.

[5] P. Misra, B. P. Burke, and M. M. Pratt, "GPS performance in navigation," Proceedings of the IEEE, vol. 87, no. 1, pp. 65-85, 1999.

[6] P. Misra and P. Enge, Global Positioning System: Signals, Measurements, and Performance, Ganga-Jamuna Press, Massachusetts, 2001.

[7] P. Bahl and V. N. Padmanabhan, "RADAR:an in-building RF-based user location and tracking system," in IEEE INFOCOM, 2000, pp. 775-784.

[8] N. Bulusu, J. Heidemann, and D. Estrin, "GPS-less low-cost outdoor localization for very small devices," IEEE Personal Communications, pp. 28-34, October 2000.

[9] L. Doherty, K. S. J. Pister, and L. E. Ghaoui, “Convex position estimation in wireless sensor networks," in IEEE INFOCOM, 2001, pp. 1655-1663. 
[10] J. Hightower and G. Borriello, "Location systems for ubiquitous computing," Computer, vol. 34, no. 8, pp. 57-66, August 2001.

[11] B. Karp, Geographic Routing for Wireless Networks, Ph.D. thesis, Harvard University, 2000.

[12] H. Takagi and L. Kleinrock, "Optimal transmission range for randomly distributed packet radio terminals," IEEE Transactions on Communications, vol. 32, no. 3, pp. 246-257, 1984.

[13] G. Finn, "Routing and addressing problems in large metropolitan-scale internetworks,” Technical Report ISI/RR-87-180, USC/ISI, March 1987.

[14] T. C. Hou and V. O. K. Li, "Transmission range control in multihop packet radio networks," IEEE Transactions on Communications, vol. 34, no. 1, pp. 38-44, 1986.

[15] E. Kranakis, H. Singh, and J. Urrutia, "Compass routing on geometric networks," in the 11th Canadian Conference on Computational Geometry, Vancouver, Canada, August 1999.

[16] I. Stojmenovic and X. Lin, "Loop-free hybrid single-path/flooding routing algorithms with guaranteed delivery for wireless networks," IEEE Transactions on Parallel and Distributed Systems, vol. 12, no. 10, pp. 246-257, 2001 .

[17] P. Bose, P. Morin, I. Stojmenovic, and J. Urrutia, "Routing with guaranteed delivery in ad hoc wireless networks," ACM Wireless Networks, vol. 7, no. 6, pp. 609-616, November 2001.

[18] B. Karp and H. T. Kung, "GPSR : greedy perimeter stateless routing for wireless networks," in ACM MobiCom, 2000, pp. 243-254.

[19] Y. B. Ko and N. H. Vaidya, "Location-aided routing (LAR) in mobile ad hoc networks," ACM/Baltzer Wireless Networks (WINET) Journal, vol. 6, no. 4, pp. 307-321, 2000.

[20] D. Son, A. Helmy, and B. Krishnamachari, "The effect of mobility-induced location errors on geographic routing in ad hoc networks: Analysis and improvement using mobility prediction," in IEEE Wireless Communications and Networking Conference (WCNC), March 2004. 
[21] S. Slijepcevic, S. Megerian, and M. Potkonjak, "Location errors in wireless embedded sensor networks: Sources, models, and effects on applications," ACM SIGMOBILE Mobile Computing and Communications Review, vol. 6, no. 3, pp. 67-78, 2002.

[22] Y. Kim, J. Lee, and A. Helmy, "Impact of location inconsistencies on geographic routing in wireless networks," in ACM International Workshop on Modeling, Analysis and Simulation of Wireless and Mobile Systems (MSWiM), 2003, pp. 124-127.

[23] R. Durrett, Probability: Theory and Examples, Duxbury Press, Belmont, CA, 2nd edition, 1996.

[24] T. He, C. Huang, B. M. Blum, J. A. Stankovic, and T. Abdelzaher, "Rangefree localization schemes for large scale sensor network," in ACM MobiCom, 2003, pp. 81-95.

[25] A. Paoulis and S. U. Pillai, Probability, Random Variables, and Stochastic Process, McGraw-Hill, New York, 4th edition, 2002.

[26] A. Davies, "An overview of bluetooth wireless technology and some competing lan standards," in the 1st IEEE International Conference on Circuits and Systems for Communications, St Petersburg, Russia, June 2002, pp. 206-211.

[27] M. Russell I. Stojmenovic and B. Vukojevic, "Depth first search and location based localized routing and qos routing in wirless networks," Computers and Informatics, vol. 21, no. 2, pp. 149-165, 2002. 